\title{
Low initial aspect-ratio direct-drive target designs for shock- or self-ignition in the context of the laser Megajoule
}

\author{
V. Brandon ${ }^{1}$, B. Canaud ${ }^{1}$, M. Temporal ${ }^{2}$ and R. Ramis ${ }^{3}$ \\ ${ }^{1}$ CEA, DIF, F-91297 Arpajon, France \\ ${ }^{2}$ Centre de Mathématiques et de Leurs Applications, ENS Cachan and CNRS, 61 avenue du \\ President Wilson, F-94235 Cachan, Cedex, France \\ ${ }^{3}$ ETSIA, Universidad Politécnica de Madrid, 28040 Madrid, Spain \\ E-mail: benoit.canaud@cea.fr
}

\begin{abstract}
Analysis of low initial aspect ratio direct-drive target designs is carried out by varying the implosion velocity and the fuel mass. Starting from two different spherical targets with a given $300 \mu \mathrm{g}$-DT mass, optimization of laser pulse and drive power allows to obtain a set of target seeds referenced by their peak implosion velocities and initial aspect ratio $(A=3$ and $A=5)$. Self-ignition is achieved with higher implosion velocity for $A=5$-design than for $A=3$-design. Then, rescaling is done to extend the set of designs to a huge amount of mass, peak kinetic energies and peak areal densities. Self-ignition kinetic energy threshold $E_{k}$ is characterized by a dependance of $E_{k} \sim v^{\beta}$ with $\beta$-values which depart from self-ignition models. Nevertheless, self-ignition energy is seen lower for smaller initial aspect ratio. An analysis of Two-Plasmons Decay threshold and Rayleigh-Taylor instability e-folding is carried out and it is shown that two-plasmon decay threshold is always overpassed for all designs. The hydrodynamic stability analysis is performed by embedded models to deal with linear and non-linear regime. It is found that the $A=5$-designs are always at the limit of disruption of the shell.
\end{abstract}

Keywords: direct drive, LMJ, inertial confinement fusion, target design

\section{Introduction}

Thermonuclear fusion of light elements [1] is one of the main challenge of the scientific community as an alternative for electrical power plants $[2,3]$. While developing country effort focuses on magnetic fusion, Inertial Confinement Fusion (ICF) begins to be looked at as another promising candidate and fusion reactors projects are undertaken in the United States with the Laser Inertial Fusion Energy (LIFE) $[4,5]$ or in Europe with the High Power laser Energy Resaerch (HiPER) [6]. As the Inertial Fusion Energy (IFE) concept requires specifically high thermonuclear gain, a proof-of-principle of ICF-produced thermonuclear ignition has still to be done and could be demonstrated on the National Ignition Facility (NIF) [7] in the US or on the future Laser MegaJoule (LMJ) [8] in France.

In the principle, ICF [9] consists in imploding a spherical capsule of Deuterium Tritium (DT) mixture at very high implosion velocities by irradiating its external side with sufficiently intense radiation. Two different ways are considered, the first one with intense laser beams, named Direct Drive (DD), and the second one using the quasi-isotropic $x$-rays created by the laser irradiation of the interior of a small cavity (hohlraum), named Indirect Drive (ID).

NIF and LMJ are ID-designed facilities [7, 10,11] and planed to provide moderate thermonuclear gains $[12,13]$.

Nevertheless, direct-drive fusion is still an option $[14,15]$ studied for both facilities as Polar Direct Drive (PDD) [16, 17] for NIF or basic direct drive for LMJ $[18,19]$. The last should need zooming technique [20], first proposed by the Naval Research Laboratory (NRL) [21] for KrF laser fusion and widely used in their DD-target designs [22-24], to achieve self-ignited high-gain thermonuclear fusion. Zooming should take benefits of the intrinsic multi-beam quad-structure of LMJ [20, 25]. Without zooming, the direct drive LMJ fusion should be marginally igniting [19].

Alternative ICF schemes exist to produce high thermonuclear yields, such as jet ignition [26], Impact Ignition (ImI) $[27,28]$, or Fast Ignition (FI) $[29,30]$ as for the HiPER project $[31,32]$. They are mainly based on a two-separate step approach, the first being the implosion of the capsule, currently named the fuel assembly [33-35], and the second being ignition of thermonuclear fusion reactions [36]. 
Recently, a new approach was proposed by the University of Rochester (UR-LLE), named Shock-Ignition (SI) [37]. It consists in launching through a pre-compressed fuel an additional strong converging shock wave that creates non-isobaric conditions of ignition. Such an approach has been shown to reduce the ignition conditions [38]. The principle is so simple and promising that it has been widely used by a part of our scientific community for their own ICF designs [38-41]. Indeed, for assumption, HiPER FI-target has been shock ignited [39] by direct numerical simulations and its robustness [39] and modeling [42] were analyzed. Specifically, Shock-Ignition appears to improve the target stability [43] during the deceleration phase of direct-drive implosion that suffers Rayleigh-Taylor instability at the hot-spot surface [44-47]. NRL has also addressed the interest of shock-ignition for the NIKE $\mathrm{KrF}$ laser project for sub-megajoule designs [3, 24, 41]. For NIF- [40] and LMJ-class [38] facilities, high thermonuclear gains are expected in direct drive approach. While NIF could mainly use the PDD $[16,17]$, DD studies, in the context of LMJ, have exhibited the importance of illumination uniformity [48-50] to warrant fuel assembly sphericity in order to reduce ignitor spike powers [51]. Indeed strong low mode asymmetries could lead to employ very intense ignitor spike powers. Such aspect leads also to strong requirement for the optimization of PDD with LMJ [50]. It has been shown also that the choice of a target design is crucial for the ignitor spike [38]. For instance, for the LMJ, a HiPER-like target design was used to address shock ignition feasibility and characteristics but the low laser-target coupling efficiency due to all-DT ablator appeared to be inefficient for direct-drive fusion on LMJ.

At this step, we felt the necessity to look at target design to address the relationship between self-ignition threshold, implosion velocity and target design in the context of fuel assembly and shock ignition. Indeed, a low-implosion velocity should lead to high self-ignition threshold. As already shown $[38,39]$, when the design is far from its self-ignition kinetic energy, the spike power needed for ignition becomes well above $200 \mathrm{TW}$ and thus intensities on target can trigger parametric instabilities [52,53], generates hot-electrons for which the transport can become a key issue for SI $[39,52,54]$ and laser absorption could be reduced. For marginally igniting target, below and close to the self-ignition threshold, ignitor spike powers are reduced [38] and intensities can be close to parametric instabilities thresholds [51]. Such trade-off between reduced implosion velocity and ignition requirement leads us to design new targets that could meet both requirements and offer the opportunity to achieve tunable thermonuclear moderate gain on LMJ, as predicted for noncryogenic double-shell designs [55].

The first consideration developed here concerns the ablator material. As we know since few years [56], the 'All-DT' ablator has a very poor laser absorption rate. The use of carbon-dopant in DT-wetted foam [15,56-59] can significantly improve the laser-target efficiency. However, such foam could have deleterious effects [60] on the target implosion due to shock mistiming. Indeed, the heterogeneous aspect of the foam can increase the first shock velocity [60-64] during the target implosion due to baroclinic deposition when the shock travels through the foam [65-68]. In addition,
3D-vorticity fields and density fluctuations in post-shock medium could alter the propagation of secondary shock waves [69-72] launched during the ramp-up of the laser pulse. Such uncertainties concerning the first shock propagation can be removed by employing a pure $\mathrm{CH}$ ablator. The price is a risk for the hydrodynamic stability during the acceleration phase [73-76]. Such problem should be significantly reduced by means of adiabat shaping [77-80] or by adding radiative dopant in order to create a double ablation front $[81,82]$. However, this is not the aim of this paper and we will consider only the simple case of a $\mathrm{CH}$-dense ablator. these stabilizing processes chould be addressed separately and do not alter the conclusion of the work presented here.

In principle, it is possible to improve the hydrodynamic stability of the implosion by acting directly on the initial aspect ratio of the target and on the implosion velocity. A reduction of both parameter compared to conventional baseline designs $[19,83]$, improves the stability. However, this way has strong effect on the thermonuclear fusion gain and the kinetic selfignition threshold.

Here, we address two distinct aspect ratios and different implosion velocities. The goal is to obtain a huge variety of designs which could be considered for self-ignition, as in baseline high-gain direct drive fusion, or for shock ignition, below the self-ignition threshold. Moreover, if a self-igniting DD design fails to ignite on LMJ, we should consider additional ignition capability with shock ignition. In this way, we need to know how far from the self-ignition threshold, the design is and what is the safety or kinetic margin.

The results presented here are separated in the following parts. A first section addresses the design's parents: for a given DT-shell mass, a target and a laser pulse shape for which implosion characteristics (peak velocities, peak areal densities) are given. Then, parametric and hydrodynamic instabilities are addressed for each design. In the next section, a scaling is done for every implosion velocities and initial aspect ratios. This allows to characterize the self-ignition kinetic threshold for each seed (referenced by the peak implosion velocity and the initial aspect ratio). In the last section, parametric and hydrodynamic instabilities are studied in the context of selected target designs. Two-plasmon decay instability (TPD) $[84,85]$ is seen to be always over-pasted during the drive part of the laser pulse. Rayleigh-Taylor Instability (RTI) $[86,87]$ is estimated in the linear and nonlinear regime by the way of existing models [74-76,88].

Calculations are performed using the one-dimensional (1D) Lagrangian radiation-hydrodynamics code FCI 1D [89]. It includes tabulated equations of state (EoS) (SESAME [90], we have not taken into account the new Caillabet's EoS $[91,92]$, that is not essential here.), flux-limited Spitzer-Harm heat transport (here the flux limiter is set at $6 \%$ ), multi-group radiative transfer, multi-group alpha-particle transport, and neutron transport. Laser absorption is done by inverse bremsstrahlung along 1 D-incoming radially rays without refraction or reflection. Degeneracy of the fuel during the deceleration is taken into account only in the thermal conductivity using a harmonic average between Spitzer and Hubbard [93] model that is validated by quantum molecular dynamics calculations [94]. 


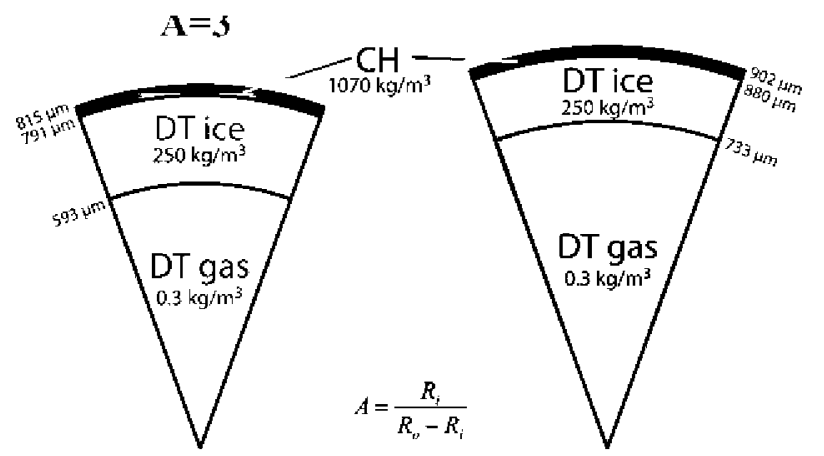

Figure 1. $300 \mu \mathrm{g}$-DT Target design with a CH-mass of $208 \mu \mathrm{g}$ and $235 \mu \mathrm{g}$ for $A=3$ (left) and $A=5$ (right) respectively.

\section{Genesis of target families}

Spherical cryogenic-DT capsules coated by a $\mathrm{CH}$-ablator layer, enclosing DT gas are considered. Target sizes are given by fixing the large amount of $300 \mu \mathrm{g}$-fuel mass and both initial aspect ratios $\mathrm{A}=\mathrm{R}_{\text {in }} /\left(\mathrm{R}_{\text {ext }}^{\mathrm{DT}}-\mathrm{R}_{\text {in }}^{\mathrm{DT}}\right)=3$ and $A=5$ where $\mathrm{R}_{\text {ext }}^{\mathrm{DT}}$ and $R_{\text {in }}^{\mathrm{DT}}$ stand for the external and inner radii of the payload DT layer. This determines the sizes of the DT shell. This mass of DT fuel is expected to produce a fusion yield of order of magnitude of $30 \mathrm{MJ}$ for a burn-up fraction of $f \sim 30 \%$. Such an initial aspect ratio is lower than baseline direct drive ones such as HiPER target [31] or direct-drive target for LMJ [58] or NIF [83] that are close to $A=7$. Low initial aspect ratios have been chosen because, in principle, they are supposed to be more hydrodynamicaly stable during the implosion.

The CH-ablator thickness depends directly on the desired implosion velocity and the drive power we expect to use. To estimate the thickness of the CH-ablator, we begin by Lindl's scaling laws [9]:

$$
\begin{gathered}
p_{a}(\mathrm{~Pa})=4 \times 10^{-2}\left(\frac{I\left(\mathrm{~W} \mathrm{~cm}^{-2}\right)}{\lambda(\mathrm{m})}\right)^{\frac{2}{3}} \\
\dot{m}_{a}\left(\mathrm{~kg} \mathrm{~m}^{-2} \mathrm{~s}^{-1}\right)=2.6 \times 10^{-7}\left(\frac{I\left(\mathrm{~W} \mathrm{~cm}^{-2}\right)}{\lambda^{4}(\mathrm{~m})}\right)^{\frac{1}{3}}
\end{gathered}
$$

where $I$ is the laser intensity, and $\lambda$ the laser wavelength. Considering the Rocket's effect as the main engine of the implosion, the implosion velocity reads as:

$$
\mathrm{v}=\frac{p_{a}}{\dot{m}_{a}} \ln \left(\frac{m_{0}}{m_{\mathrm{sh}}}\right)
$$

where $m_{0}$ is the initial shell mass and $m_{\mathrm{sh}}$ is the sum of the payload mass of DT and a residual small amount of $\mathrm{CH}$.

Using the previous equations and a DT-ice mass of $300 \mu \mathrm{g}$ leads to the targets sketched on figure 1 .

The implosion is driven in a two distinct parts: the acceleration stage and the deceleration phase. In the first stage, the shell is accelerated to the desired implosion velocity without increasing the internal energy above a limit that is characterize by the in-flight adiabat of the fuel defined by $\alpha_{\text {if }}=P_{\text {shell }} / P_{\text {Fermi }}$ where $P_{\text {shell }}$ and $P_{\text {Fermi }}[\mathrm{MPa}]=2.17 \varrho^{5 / 3}\left[\mathrm{~kg} \mathrm{~m}^{-3}\right]$ are the shell and Fermi pressure respectively. To keep internal energy in the shell at

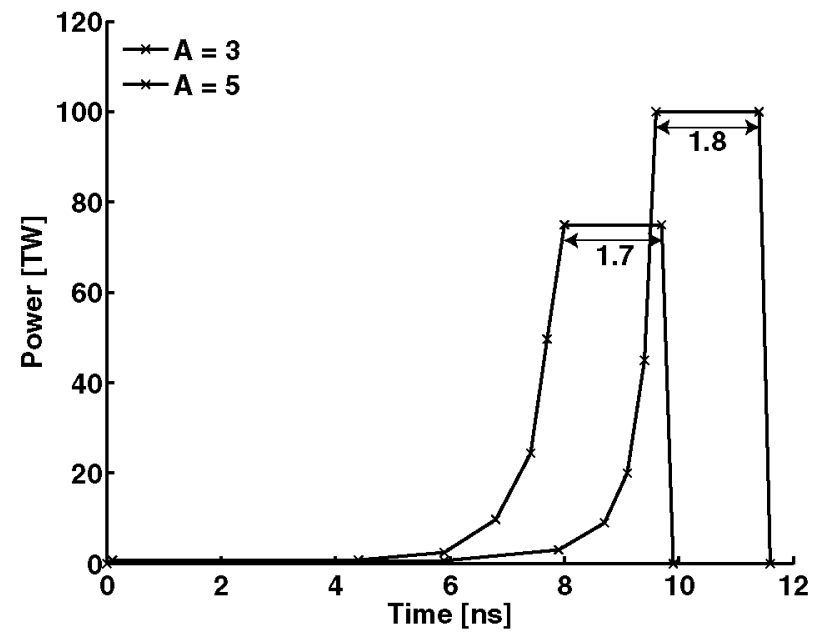

Figure 2. Initial laser pulse shape for $A=5$ (yellow) with $1.7 \mathrm{~ns}$ drive duration, and $A=3$ (green) with $1.8 \mathrm{~ns}$ drive duration.

a chosen level, during the acceleration, time-resolved external pressure law has to ramp-up shockless following the Kidderlike law [95]. Nevertheless, laser-driven implosion cannot be done shockless and the laser pulse will always launch few shocks through the DT-shell. Thus to keep the shell on the desired adiabat, the laser pulse is shaped in three distinct parts: the foot, that launches a first shock placing the DT on the desired adiabat, a Kidder-like ramp, and a drive part that gives the desired implosion velocity. In our designs, we choose an adiabat $\alpha_{\text {if }}=1.2$, produced by a 300 GPa-first shock. Such internal energy level is at a crossing point of Hugoniot curves determined by SESAME EoS and Caillabet's EoS [91] and reduces the effect of changing EoS during the acceleration phase [92]. The implosion velocity is chosen at an arbitrary value of $300 \mathrm{~km} \mathrm{~s}^{-1}$ thus the 1D-laser power and duration of drive are $100 \mathrm{TW}$ and $1.8 \mathrm{~ns}$ ( $80 \mathrm{TW}$ and $1.7 \mathrm{~ns}$ ) for the $A=3$ $(A=5)$.

Laser pulse shapes, described in figure 2, are finally numericaly determined, in 1D, by adjusting the ramp-up in order to control the multiple-shock timing to break through the inner side of DT ice with a temporal delay of $300 \mathrm{ps}$.

Finally, the total 1D-incident laser energy is $230 \mathrm{~kJ}$ and $200 \mathrm{~kJ}$ for the $A=3$ and $A=5$ design, respectively

A variation of laser shape is done to scan an interval of implosion velocities by modifying the drive power and the laser ramp. A previous study [96], using a random perturbation of the ramp, has shown that the Kidder's law could be optimized. This approach was used to refine the laser pulse shape, optimizing the thermonuclear energy and the peak areal density. The peak implosion velocity v, defined as the maximum during implosion of the velocity calculated from the average DT-shell kinetic energy, varies between $260 \mathrm{~km} \mathrm{~s}^{-1}$ and $360 \mathrm{~km} \mathrm{~s}^{-1}$. The optimization required few thousands of computations for each initial aspect ratio. Variations of thermonuclear energies as a function of the peak implosion velocity are given in figure 3 for $A=3$ and $A=5$. When the implosion velocity is sufficiently high, the kinetic energy $E_{k}$ becomes greater than the self-ignition kinetic energy threshold, ignition occurs, and thermonuclear energy growths asymptotically to $27 \mathrm{MJ}$ for both aspect ratios. We can see that 


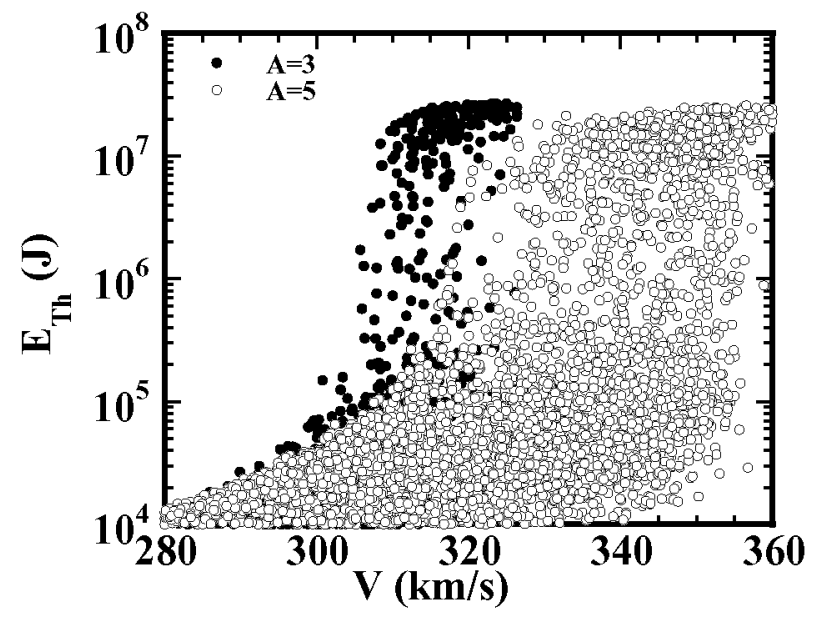

Figure 3. Thermonuclear energy versus implosion velocity for $A=3$ (filled circles) and $A=5$ (open circles).

self-ignition threshold is reached at lower implosion velocity for $A=3\left(v=306-308 \mathrm{~km} \mathrm{~s}^{-1}\right)$ than for $A=5(v=317$ $\left.329 \mathrm{~km} \mathrm{~s}^{-1}\right)$ as predicted by model developed in [97]. Below the thresholds, targets are marginally igniting but they can be possibly shock-, fast- or impact-ignited.

We also notice that absorbed energy needed to reach implosion velocity of self-ignition threshold and drive powers are higher for lower initial aspect ratio $\left(A=3, E_{a}=234\right.$ $250 \mathrm{~kJ}$, and $\left.A=5, E_{a}=219-226 \mathrm{~kJ}\right)$. At the opposite, peak areal densities are higher for $A=3$ at same implosion velocities.

Few designs are selected at the upper frontier of the data clouds for both A. These designs, summarized in the table 1, produce the maximum thermonuclear energy whatever the absorbed energy is for different implosion velocities. In this table $1, \mathrm{v}, \mathrm{P}_{d}, \mathrm{E}_{a}, \varrho \mathrm{R}$ and $\mathrm{E}_{\text {th }}$ stand for peak implosion velocity, laser drive power, absorbed energy, peak areal density, and thermonuclear energy, respectively.

We observed that the areal densities increases slightly for $A=3$ when the implosion velocity increases while for $A=5$, we observe a stronger dependence with $\mathrm{v}$ peak. The last is imploded with a fixed drive duration and the optimization results have indicated that a larger duration leads to a higher peak areal density [96].

Scaling laws existing in literature $[33,34]$, written as $\varrho \mathrm{R}\left[\mathrm{kg} \mathrm{m}^{-2}\right]=12 / \alpha_{\mathrm{if}, \text { min }}^{0.54}\left(E_{a}[\mathrm{~kJ}] / 100\right)^{0.33}\left(\mathrm{v}\left[\mathrm{km} \mathrm{s}^{-1}\right] /\right.$ $300)^{0.06}$, predict a peak areal density evolving from $14 \mathrm{~kg} \mathrm{~m}^{-2}$ to $17 \mathrm{~kg} \mathrm{~m}^{-2}$ for both $\mathrm{A}$. Here, $\alpha_{\mathrm{if}, \min }=1$ is chosen in accordance with the calculation as the minimum numerical value of in-flight adiabat in the compressed DT-shell deduced from the classical formula $\alpha=P_{\text {shell }} / P_{\text {fermi }}$. Here, we have used the absorbed laser energy whilst in [34], the incoming laser is used. We can correct this by considering that the integrated laser-target coupling efficiency, defined as $E_{a} / E_{\text {incident }}^{3 \mathrm{D}} \sim 0.5$. Thus the model leads to the areal densities: $(\varrho R)_{\text {peak }}^{\text {mode }}\left[\mathrm{kg} \mathrm{m}^{-2}\right] \in[17,19]$. By using this correction we get a better agreement with numerical results for only $A=3$. In fact, the model suggests no relevant effect of the initial aspect ratio, in contradiction with numerical results.

Hydrodynamic efficiency $\eta=E_{k} / E_{a}$, referred as the ratio of peak shell kinetic energy over the total laser absorbed energy is seen varying very slowly with peak implosion velocity both for $\mathrm{A}$. We notice that $\eta_{A=3} \in[0.055,0.06]$ and $\eta_{A=5} \in[0.069,0.073]$ in the range of velocity. Pioneering works $[33,34]$ have determined the following scaling law: $\eta \simeq(0.05) I_{15}^{-0.25}\left(\mathrm{v}\left[\mathrm{km} \mathrm{s}^{-1}\right] / 300\right){ }^{0.75}\left(\lambda_{L}[\mu \mathrm{m}] / 0.35\right)^{-0.5}$ for target with an initial aspect ratio of $A=1.3$ [33] and $A=3$ [34]. Applied to our cases and estimating the intensity at the critical radius (by dividing the incoming laser power by the critical density surface), this scaling leads to $\eta=0.026$ for $A=3$ and $\eta=0.03$ for $A=5$ whatever the velocity is, well below our results. If we consider now another definition for intensity to be taken in the model: $I=P_{d} / 4 \pi r_{0}^{2}$, where $r_{0}$ is the initial external radius of the target, we get $\eta \sim 0.047$ for $A=3$ and $\eta \sim 0.055$ for $A=5$. This last definition improves the matching of the model with numerical results but with still a residual shift of $30 \%$.

Above the self-ignition threshold, the thermonuclear energy is asymptotically converging toward a burn-up fraction of $f \sim 30 \%$. Using the Fraley's expression [98]: $f=$ $1 /\left(1+\mathrm{H}_{\mathrm{B}} /(\varrho r)\right)$, we deduce from table $1, \mathrm{H}_{B} \sim 5$, of the same order than Fraley's estimate $\left(H_{B} \sim 6\right.$ in [98]).

To conclude this part, models existing in the literaturemismatch the peak areal density and the hydrodynamic efficiencies simultaneously for both $\mathrm{A}$, showing that the initial aspect ratio is an additional parameter that must be accounted for.

\section{Laser parametric instabilities and hydrodynamic stability analysis}

\subsection{Laser intensities and two-plasmons instability threshold}

The laser incident intensity is one parameter important for target design. Indeed, for sufficient level above threshold, it can generate parametric laser plasma instabilities that could produce hot electrons in the corona and preheat the cold fuel.

One of the most relevant parametric instabilities concerns the Two-Plasmons Decay (TPD) that corresponds to the generation of two electron plasma waves resonantly with the laser frequency at the quarter-critical density. An expression $[84,85]$ of the absolute instability threshold intensity has been proposed, in the past, and reads as $I_{2 \omega_{p}}\left[10^{14} \mathrm{~W} \mathrm{~cm}^{-2}\right]=80 T_{e}[\mathrm{keV}] \lambda^{-1}[\mu \mathrm{m}] \mathrm{L}_{n_{e}}^{-1}[\mu \mathrm{m}]$, where $\mathrm{L}_{n_{e}}$ is the gradient scale length of electronic density at $\mathrm{n}_{c} / 4$ : $\mathrm{L}_{n_{c} / 4}=\mathrm{n}_{e} / \nabla \mathrm{n}_{e}$. Electron density gradient scale lengths are strongly oscillating during the drive duration as shown in figure 4 whilst temperature increases sharply. These oscillations are systematically observed in all designs and come from shock and rarefaction waves traveling in the plasma, even in the plume. Averages are done over the drive pulse and are given in figure 5 . Such a procedure introduces errors of roughly $10 \%$ especially concerning the density scale length and fluctuations can appear when comparing values for different velocities as seen in figure 5. As expected, higher the implosion velocities, higher the electron temperatures are. They evolve from $4 \mathrm{keV}(3 \mathrm{keV})$ to $6 \mathrm{keV}(5 \mathrm{keV})$ when implosion velocity evolves from $260 \mathrm{~km} \mathrm{~s}^{-1}\left(280 \mathrm{~km} \mathrm{~s}^{-1}\right)$ to $320 \mathrm{~km} \mathrm{~s}^{-1}\left(360 \mathrm{~km} \mathrm{~s}^{-1}\right)$ for $A=3(A=5)$ capsule. Gradient scale lengths are greater for $A=5$ than for $A=3$. The lengths are roughly constant at $300 \mu \mathrm{m}$ for $A=3$ whilst evolve from $300 \mu \mathrm{m}$ to $370 \mu \mathrm{m}$ for $A=5$ as the implosion velocity 
Table 1. List of working points selected to compute scaled targets.

\begin{tabular}{|c|c|c|c|c|c|c|c|c|c|c|c|}
\hline \multirow[b]{2}{*}{$\begin{array}{l}\mathrm{v} \\
\mathrm{km} \mathrm{s}^{-1}\end{array}$} & \multicolumn{5}{|c|}{$A=3$} & \multicolumn{6}{|c|}{$A=5$} \\
\hline & $\begin{array}{l}\mathrm{P}_{d} \\
\mathrm{TW}\end{array}$ & $\begin{array}{l}\mathrm{E}_{a} \\
\mathrm{~kJ}\end{array}$ & $\begin{array}{l}\varrho \mathrm{R} \\
\mathrm{kg} \mathrm{m}^{-2}\end{array}$ & $\begin{array}{l}\mathrm{E}_{\text {th }} \\
\mathrm{kJ}\end{array}$ & $\eta$ & $\begin{array}{l}\mathrm{V} \\
\mathrm{km} \mathrm{s}^{-1}\end{array}$ & $\begin{array}{l}\mathrm{P}_{d} \\
\mathrm{TW}\end{array}$ & $\begin{array}{l}\mathrm{E}_{a} \\
\mathrm{~kJ}\end{array}$ & $\begin{array}{l}\varrho \mathrm{R} \\
\mathrm{kg} \mathrm{m}^{-2}\end{array}$ & $\begin{array}{l}\mathrm{E}_{\text {th }} \\
\mathrm{kJ}\end{array}$ & $\eta$ \\
\hline 260 & 74 & 174 & 16.4 & 7 & 5.98 & & & & & & \\
\hline \multirow[t]{2}{*}{270} & 79 & 188 & 17.2 & 11 & 5.95 & & & & & & \\
\hline & & & & & & 275 & 57 & 164 & 13.7 & 10.5 & 7 \\
\hline 280 & 81 & 200 & 18.1 & 14 & 5.84 & 280 & 58 & 170 & 14.2 & 13 & 6.93 \\
\hline 285 & 83 & 215 & 18.5 & 18 & 5.7 & 285 & 62 & 175 & 14.1 & 17 & 6.96 \\
\hline 290 & 100 & 215 & 18.2 & 27 & 5.85 & 290 & 63 & 179 & 14.2 & 24 & 7.07 \\
\hline 295 & 93 & 226 & 18.1 & 41 & 5.77 & 295 & 68 & 187 & 14.6 & 35 & 7.01 \\
\hline 300 & 100 & 229 & 18.0 & 150 & 5.92 & 300 & 70 & 196 & 15.1 & 65 & 6.99 \\
\hline 306 & 103 & 247 & 18.0 & 1700 & 5.67 & & & & & & \\
\hline 310 & 105 & 252 & 18.8 & 17800 & 5.74 & 310 & 75 & 205 & 15.4 & 270 & 7.12 \\
\hline 315 & 110 & 260 & 18.2 & 23000 & 5.68 & 315 & 80 & 210 & 15.4 & 600 & 7.11 \\
\hline 320 & 114 & 272 & 18.6 & 26200 & 5.63 & 320 & 83 & 216 & 15.9 & 7600 & 7.18 \\
\hline \multirow[t]{7}{*}{325} & 119 & 283 & 18.9 & 26900 & 5.6 & & & & & & \\
\hline & & & & & & 329 & 87 & 226 & 16.0 & 18119 & 7.19 \\
\hline & & & & & & 333 & 86 & 233 & 16.4 & 21411 & 7.14 \\
\hline & & & & & & 348 & 100 & 249 & 16.6 & 24862 & 7.3 \\
\hline & & & & & & 352 & 104 & 264 & 16.4 & 25064 & 7.1 \\
\hline & & & & & & 356 & 108 & 267 & 16.6 & 25695 & 7.14 \\
\hline & & & & & & 365 & 112 & 280 & 16.7 & 26296 & 7.12 \\
\hline
\end{tabular}

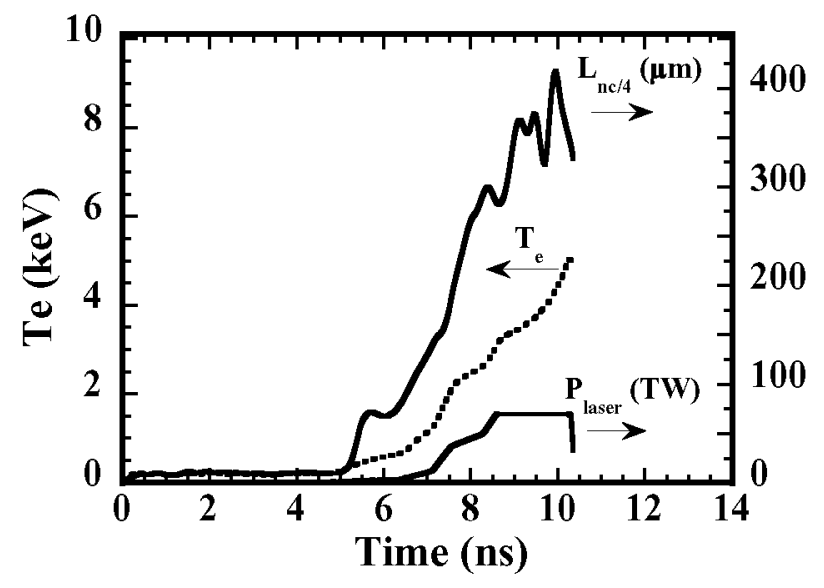

Figure 4. Time-resolved electron temperature (dotted lines) and electron density gradient length at $\mathrm{n}_{c / 4}$ (full lines) for $A=5$ and $v=300 \mathrm{~km} \mathrm{~s}^{-1}$. In the same plot is given the laser pulse.

increases. This leads to a lower TPD threshold for $A=5$ than $A=3$ as it can be seen in figure 6 .

Intensities are estimated in our calculations by dividing the incoming laser power by the surface of the quarter critical radius: $I=P_{d} /\left(4 \pi r_{n_{c} / 4}^{2}\right)$. It can be seen in figure 6 , that intensities, averaged over the drive part, are higher for $A=3$ $\left(1-2 \times 10^{15} \mathrm{~W} \mathrm{~cm}^{-2}\right)$ than for $A=5\left(5-12 \times 10^{14} \mathrm{~W} \mathrm{~cm}^{-2}\right)$. For both A, intensities overrun the TPD threshold by a factor increasing with the implosion velocity.

The ratio $\zeta=I / I_{2 \omega_{p}}$ is always higher for $A=3$ than for $A=5$ during the whole variation of the implosion velocity predicting by this way a higher sensitivity of $A=3$-designs than of $A=5$-designs. Indeed, $\zeta \in[3,3.8]$ for $A=3$ and $\zeta \in[2.5,4]$ for $A=5$ when implosion velocity $\mathrm{v}\left[\mathrm{km} \mathrm{s}^{-1}\right] \epsilon$ $[260,325]$ and $v\left[\mathrm{kms}^{-1}\right] \in[275,365]$ respectively. As the instability growths and saturates, electrons are trapped and accelerated to very high multi-keV temperatures. Recently, experimental measurement [99] of hot-electron generation by

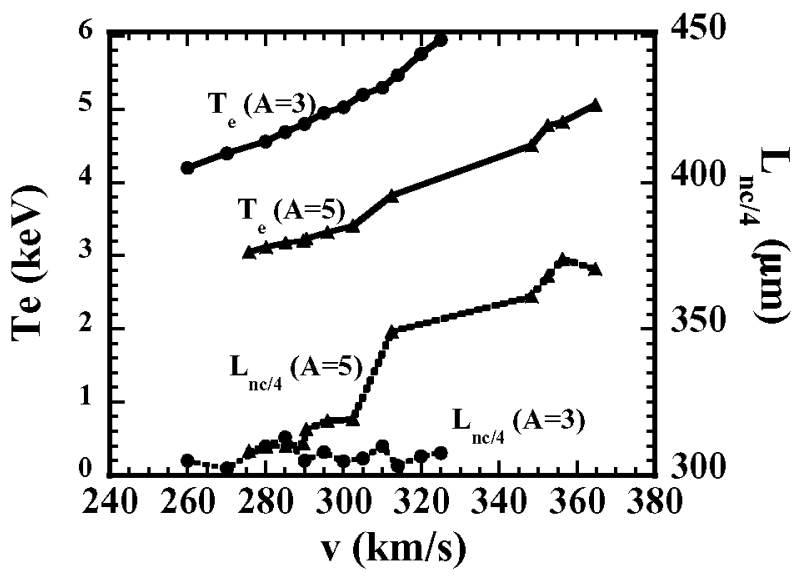

Figure 5. Average electron temperature (full lines) and electron density gradient length at $\mathrm{n}_{c / 4}$ (dotted lines) taken during the drive versus implosion velocity for $A=3$ (circles) and $A=5$ (triangles).

two-plasmon instability in Direct-Drive plasmas have been done in the range $1<\zeta \leqslant 3$. Using figure 4 of [99], the average hot-electron temperature is seen to scale as $T_{\text {hot }}[\mathrm{keV}] \sim 36(\zeta-1)$ and the energy fraction converted into hot-electron seems to saturate at $f_{\text {hot }} \sim 3-4 \%$ when $\zeta \sim 3$. These results pointed out the effect of multiple overlapping beams leading to a saturation of the fraction of hot electrons.

However, our $\zeta$ parameter is underestimated. Indeed, $\zeta$ has to be multiplied by a correcting factor which would takes into account the laser-target coupling efficiency $\tau_{\text {eff }}=$ $P_{\text {abs }} / P_{\text {incident }}^{3 \mathrm{D}} \sim 0.5$ defined as the ratio of absorbed laser power over the incident laser power (in three dimensions). The last should be larger than the absorbed power $P_{\mathrm{abs}}$ in order to deal with refraction, absorption efficiency, losses by the side of the target, and incident intensity profiles. This should lead to a $\zeta$ parameter twice the previous estimate. Thus, the predicted hot-electron temperatures, using the previous scaling laws, are in the range $T_{\text {hot }}[\mathrm{keV}] \in[180,240]$ and $T_{\text {hot }}[\mathrm{keV}] \in$ $[150,250]$ for $A=3$ and $A=5$, respectively. The 


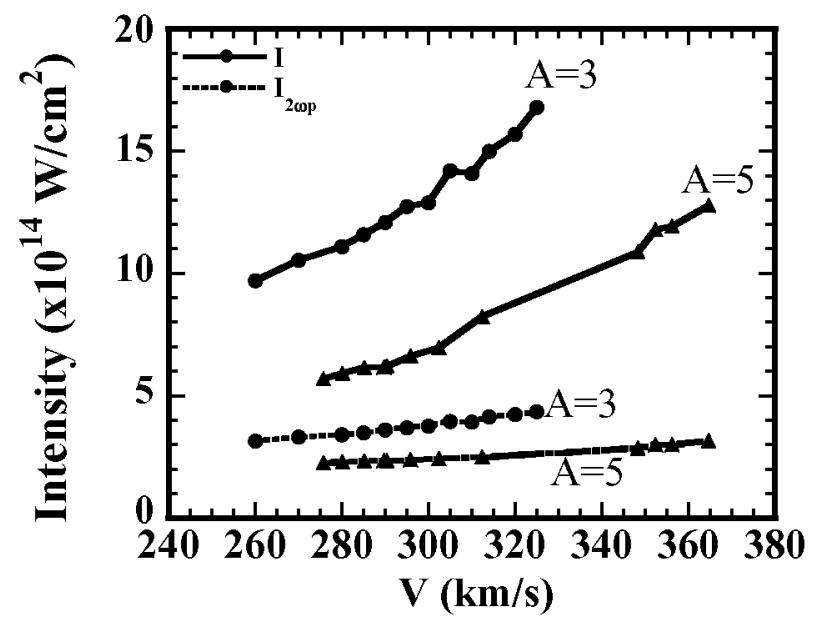

Figure 6. Average intensities (full lines) and TPD threshold (dotted lines) taken during the drive versus implosion velocity for $A=3$ (circles) and $A=5$ (triangles).

conversion fraction of laser energy into hot-electron, $f_{\text {hot }}=$ $E_{\text {hot }} / E_{\text {laser }}$, is extrapolated by fitting experimental results of literature $[99,100]$, and evolves in the range $f_{\text {hot }} \sim 4-5 \%$ for both A. Nevertheless, such an estimate is too much crude and additional experimental measurements of hot-electron in spherical plasmas relevant to direct-drive fusion are needed to validate our estimates in these regimes $(\zeta \geqslant 5)$.

\subsection{IFAR and convergence ratios}

Usually, time resolved- and peak in-flight aspect ratios (IFAR) are used to estimate the hydrodynamics stability of an implosion in ICF. Time resolved-IFAR is defined as the ratio of external capsule radius over thickness of the whole compressed shell at each time of implosion. The external radius is defined as the position of the ablation front created by laser ablation. The ablation front radius is chosen as the position of the minimum density gradient scale length. The thickness is defined as the difference between external radius and inner radius (during the whole implosion, the inner radius correspond to the interface between DT ice and inner gas). Figure 7 shows the IFAR for different velocities for both initial aspect ratios $\mathrm{A}$.

As shown on figure 7 , the time evolution of the aspect ratio depends strongly on the implosion history. Indeed, multiple shocks and rarefaction waves traveling through the shell vary its thickness. Thus, the IFAR is oscillating between peak-tovalley estimates of $20-36$ for $A=3$ and $20-56$ for $A=5$ respectively. Nevertheless, the IFAR achieves lower average as well as peak levels for $A=3$ than for $A=5$.

Hot-spot convergence ratio, $C_{h}=R_{\text {inner }}(t=0) / R_{\mathrm{hs}}$, is defined as the ratio of the initial inner shell radius over the hot-spot radius at stagnation, at the end of the implosion. $C_{h}$ is estimated for all designs.

The most noticeable effect is the confirmation of its dependence with the implosion velocity (figure 8 ). We can see that for igniting targets, $C_{h}$ is lower than for non- or marginally igniting targets due to the kinetic safety margin. Indeed, when the target design is above the self-ignition threshold, fusion reactions start before the stagnation without fusion processes.
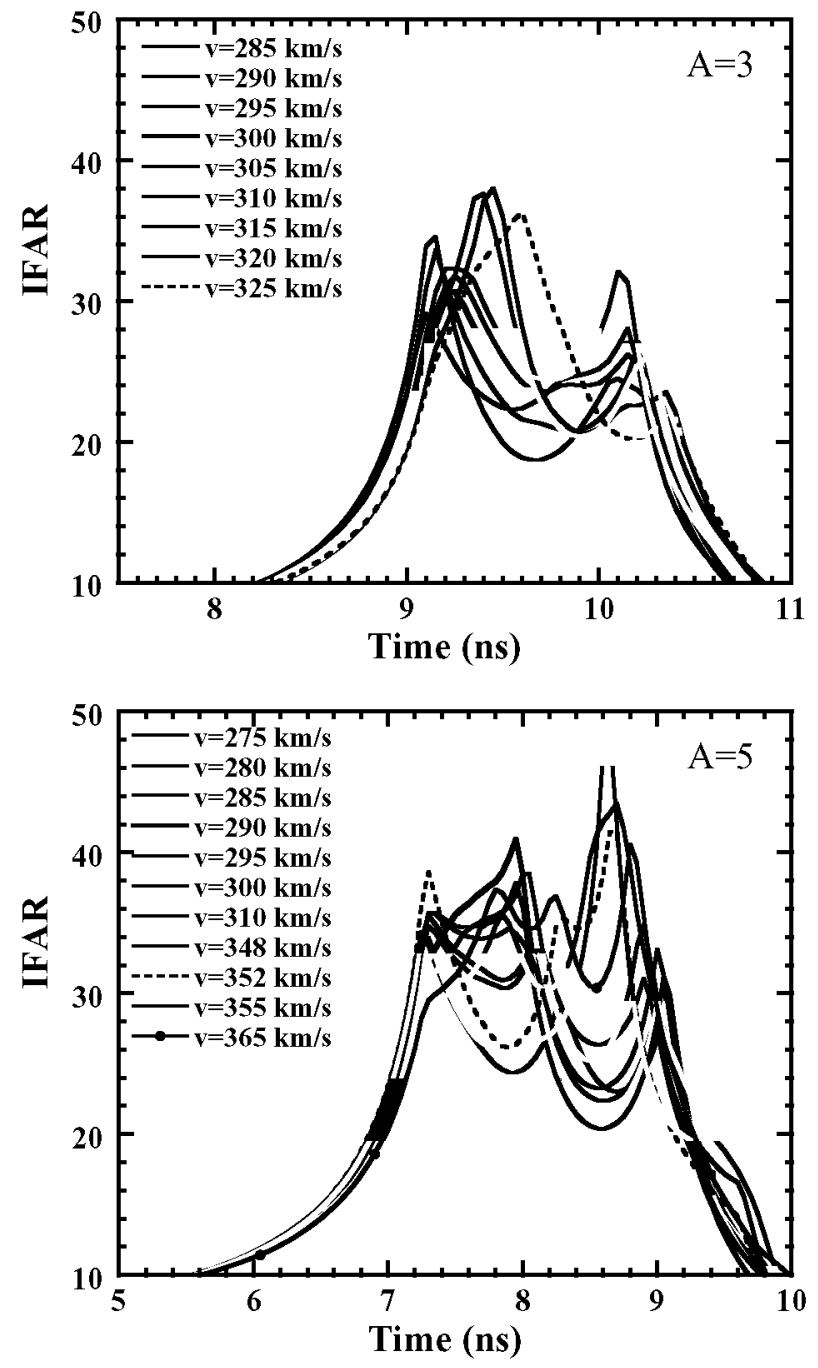

Figure 7. IFAR versus time for different implosion velocities for $A=3$ (top) and $A=5$ (bottom).

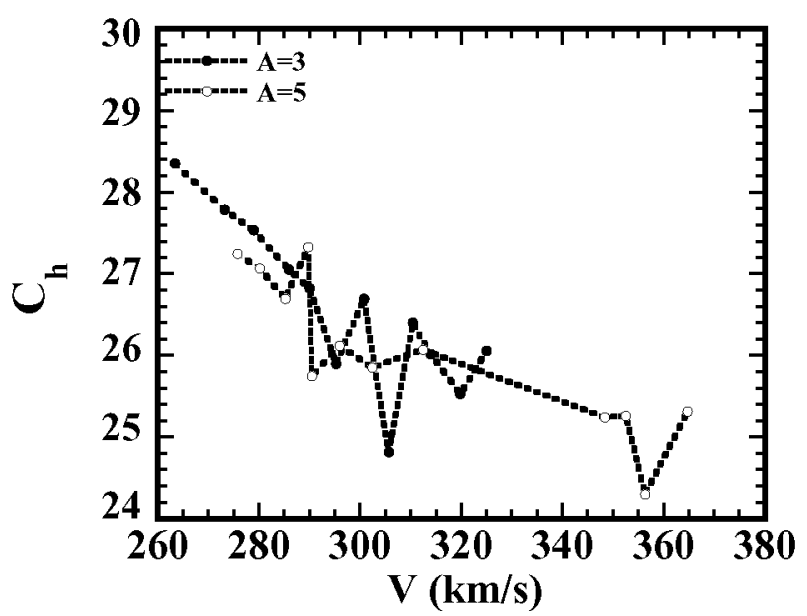

Figure 8. Hot-spot convergence ratios versus the implosion velocities for $A=3$ and $A=5$.

The self-heating induced by alpha energy deposition increases hot-spot pressure and leads to a stagnation occurring earlier with fusion processes than without. Minimum hot-spot radius, at stagnation, becomes greater when fusion processes are 
present. Thus, hot-spot convergence ratios are smaller for igniting designs. Another interesting result concerns the very tiny effect of the initial aspect ratio $\mathrm{A}$. Indeed, at the same implosion velocity, both targets have roughly the same $C_{h}$.

\subsection{High modes hydrodynamic stability of implosion}

Hydrodynamic stability of high modes is the most harmful effect on shell during implosion. Indeed, while low mode asymmetries grow slowly with the implosion and are amplified by the convergence effects, high unstable modes are strongly and quickly growing until levels that can destroy the cryogenic layer. This growth is related to the Rayleigh Taylor Instability (RTI) $[86,87]$ and takes place during the acceleration phase. We can separate the instability growth in two distinct stages. The first one corresponds to the linear growth of single modes without coupling between modes. The second one concerns the non-linear stage where modes interact and new modes are generated. The transition between both regimes is usually considered to happen when the amplitude of the single mode perturbation is of the order of one tenth the wavelength. The final external roughness of the perturbation is estimated by the use of embedded models post-processed to 1D-hydrodynamics data of the implosion. A first model is used to estimate the linear growth of the instability. Then, a saturation model is coupled to the former with the NIF's roughness spectrum as an input.

During the linear stage, the Rayleigh-Taylor instability (RTI) is stabilized by ablation processes. A first formula (Takabe's formula [73]) was proposed to estimate the RTI growth in presence of ablative stabilization few years ago and modified [101] to deals with sharp-boundary models of the ablation front [102]. The main limitations of this approach were the requirement of numerically-fitted constants that depend on the hydrodynamics data (ablator material, densities, ...). A second analysis, probably the most relevant one, was proposed later by Betti et al $[103,104]$ to express self-consistently the linear growth rate $[74,75]$, using onedimensional hydrodynamics profiles of density and pressure. The main interest of the model is that the growth rate estimate does not need a fitting procedure.

The growth rate $\gamma(t)$ is calculated directly from the $1 \mathrm{D}$ hydrodynamics time- and spatial-resolved density and pressure profiles using the formulas extracted from [75].

An example of time resolved growth rate for each initial aspect ratio at same implosion velocity of $300 \mathrm{~km} \mathrm{~s}^{-1}$ is presented on figure 9. We can see that during the first few ns of the laser pulse, RTI growth rate stays at a very low level. Then, in the drive part, the growth rate becomes very high. In addition, the growth rate aspect is different between both A. Indeed, for $A=3$, the growth rate achieves a maximum of roughly $11 \mathrm{~ns}^{-1}$ at $9.5 \mathrm{~ns}$ during about $1.5 \mathrm{~ns}$ whilst, for $A=5$, the peak value is around $8 \mathrm{~ns}^{-1}$ at $7 \mathrm{~ns}$ during $2.5 \mathrm{~ns}$. The mode-number cut-off is very high, greater than $10^{4}$. The first signal, early in time at around $2 \mathrm{~ns}$, is due to the first shock breakthrough from the $\mathrm{CH}$-ablator in the DT ice. A rarefaction wave turns back towards the shocked ablator up to the ablation front under the ablation pressure. When the rarefaction wave arrives at the ablation front, the acceleration begins and the ablation front becomes unstable at moderate level (growth rate $\sim 3 \mathrm{~ns}^{-1}$ ) during less than $1 \mathrm{~ns}$.
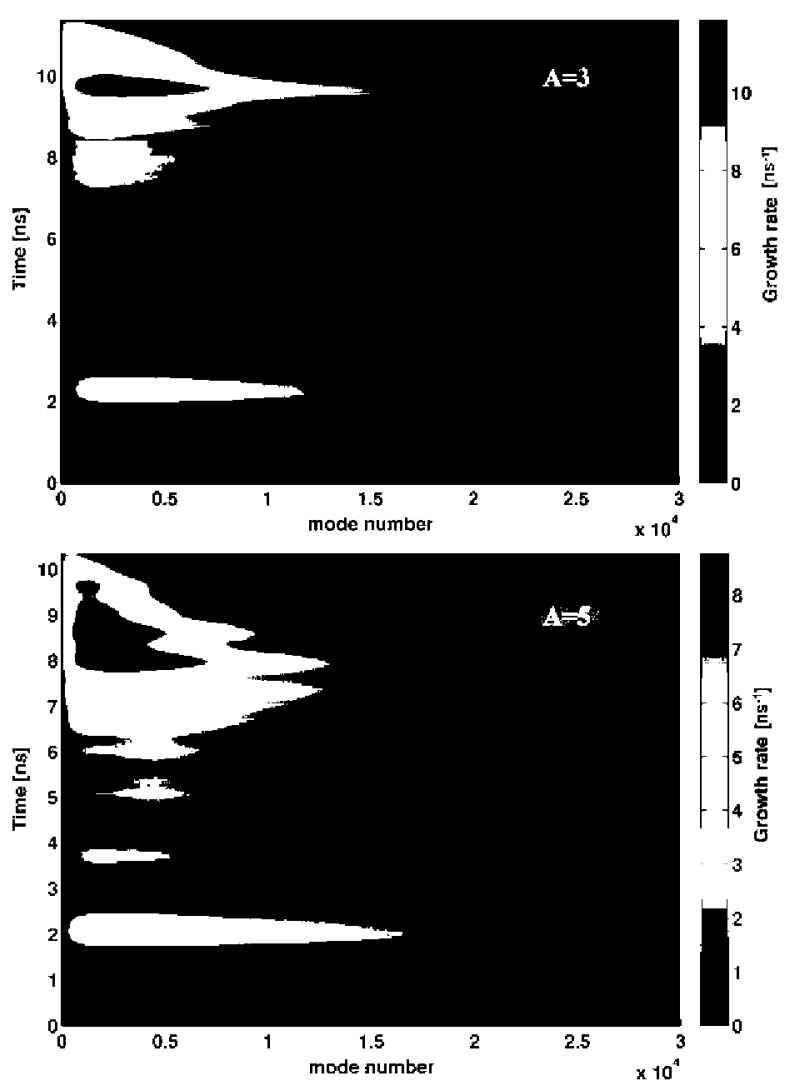

Figure 9. Time-resolved growth rates versus mode number for $A=3$ (Top) and $A=5$ (bottom), and $v=300 \mathrm{~km} \mathrm{~s}^{-1}$.

These time-resolved growth rates are estimated for each target of table 1 and for both $\mathrm{A}$ and used for the non-linear stage model. The last, based on S. Haan approach [88], allows to estimate the final roughness and the risk of shell breaking.

The time-resolved amplification of RTI modes is given by: $A_{l}(t) / A_{l 0}=\exp \left(\int_{0}^{t} \gamma\left(t^{\prime}\right) \mathrm{d} t^{\prime}\right)$.

The initial perturbation $A_{l 0}$ is the measured roughness Legendre $l$-mode number spectrum of the $\mathrm{CH}$-ablator [105]. It is characterized by a total rms rugosity of $155 \mathrm{~nm}$ for $l=2-$ 1000 , and distributed as follows: $\sigma_{2}=135 \mathrm{~nm}, \sigma_{3-100}=$ $75 \mathrm{~nm}$ (The rugosity is calculated for $l \in[3,100]: \sigma_{3-100}=$ $\sqrt{\sum_{l=3}^{100} \sigma_{l}^{2}}$, and $\sigma_{101-1000}=3.12 \mathrm{~nm}$. The amplitude spectrum is approximated by a half-NOVA -like roughness spectrum: $A_{l 0}(\mu m)=\frac{1}{2} \frac{0.19}{(l / 4)^{4}+(l / 60)^{0.4}}+\frac{1}{2} \frac{0.12 \times 10^{-3}}{(l / 60)^{0.8}+(l / 1200)^{8}}$. The amplitude of saturation is defined in Haan's model by: $A_{\text {sat }}(t)=2 r_{a}(t) / l^{2}$ where $r_{a}(t)$ is the ablation front radius. The amplitude of deformation is calculated as following:

$$
\begin{cases}A_{l}(t)=A_{l i n}(t)=A_{l 0} \mathrm{e}_{0}^{t} \gamma\left(t^{\prime}\right) \mathrm{d} t^{\prime} & \text { for } A_{l}(t)<A_{\mathrm{sat}}(t) \\ A_{l}(t)=A_{\mathrm{sat}}(t)\left(1+\ln \frac{A_{\text {lin }}(t)}{A_{\mathrm{sat}}(t)}\right) & \text { for } A_{l}(t)>A_{\mathrm{sat}}(t)\end{cases}
$$

The results are shown in figure 10 , and refer to the maximum amplitude of the perturbation, which is achieved just before the end of the laser pulse and the beginning of the deceleration. Surprisingly, the total amplification is very similar for both $\mathrm{A}$. Then, the time-resolved root-meansquare (rms) rugosity given by: $\sigma_{\mathrm{rms}}(t)=\left(\sum_{l} \frac{2 l+1}{4 \pi} A_{l}(t)^{2}\right)^{\frac{1}{2}}$ 


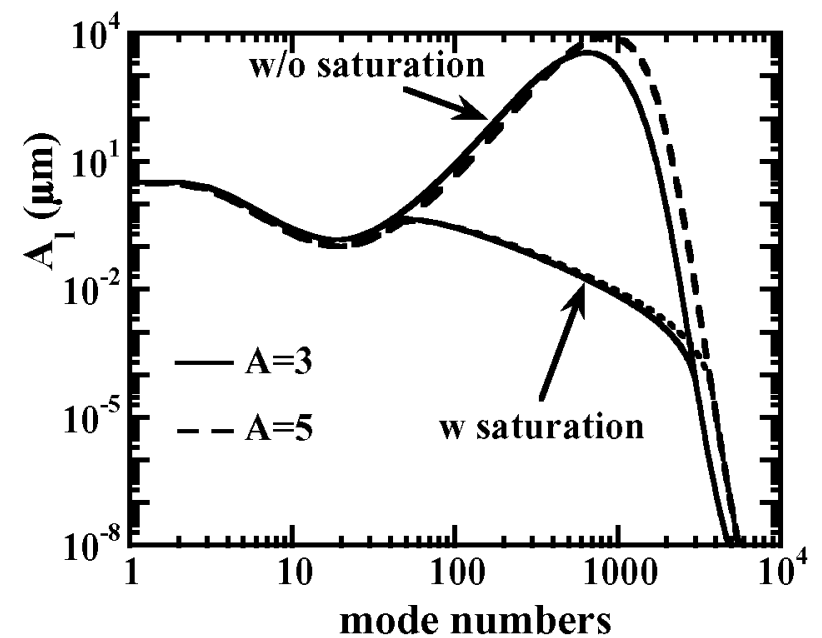

Figure 10. Total amplification versus mode numbers without (w/o) and with (w) saturation for $A=3$ (plain lines) and $A=5$ (dashed lines) at the end of acceleration phase. Here, $v=300 \mathrm{~km} \mathrm{~s}^{-1}$

is estimated for both A and compared to the target thickness $e_{\text {target }}$, defined as the difference between inner and ablation front radii. In figure 11 , the ratio $\sigma_{\text {rms }} / e_{\text {target }}$ is plotted for both A and the case $v=300 \mathrm{~km} \mathrm{~s}^{-1}$. When the ratio $\sigma_{\mathrm{rms}} / e_{\text {target }}$ becomes greater than one, the shell is broken by the spikes and bubbles induced by the instability during the drive when the shell is strongly accelerated. We observe that the $A=3$ design is more robust to the deleterious effect of RTI than $A=5$-design.

Now, we consider all the designs described in table 1. When the implosion velocity is varying, the ratio $\sigma_{\mathrm{rms}} / e_{\text {target }}$ increases above one for the $A=5$-design while, as expected, it stands below one for $A=3$.

In conclusion of the section 3 , the $A=3$-design is less robust regarding the $2 \omega_{p}$ instability and could be more preheated by hot electrons than the $A=5$-design. On another hand, the $A=5$-design is less robust regarding the RTI instability and the final rugosity achieved at the end of the acceleration should be more deleterious.

\section{Scaling laws and target design families}

The parent designs presented in table 1 are rescaled in order to build different families of designs. Following the mass rescaling pointed out by Basko et al [106] and mentioned by Murakami in his Lie group analysis [107] as a part of a more general similitude theory $[108,109]$, modifications of target sizes, time durations, energies, masses and powers are given by:

$$
\begin{gathered}
r^{\prime} \rightarrow \xi r, t^{\prime} \rightarrow \xi t, \\
E^{\prime} \rightarrow \xi^{3} E, M^{\prime} \rightarrow \xi^{3} M, \\
P \rightarrow \xi^{2} P^{\prime},
\end{gathered}
$$

where $\xi$ is the scaling factor whilst implosion velocities, In-Flight Aspect Ratio (IFAR), densities, pressures and adiabat are invariant by the scaling.

Calculations are performed for each whole implosion of scaled design. Figure 12 represents the thermonuclear energy as a function of the kinetic energy for each initial aspect ratio.

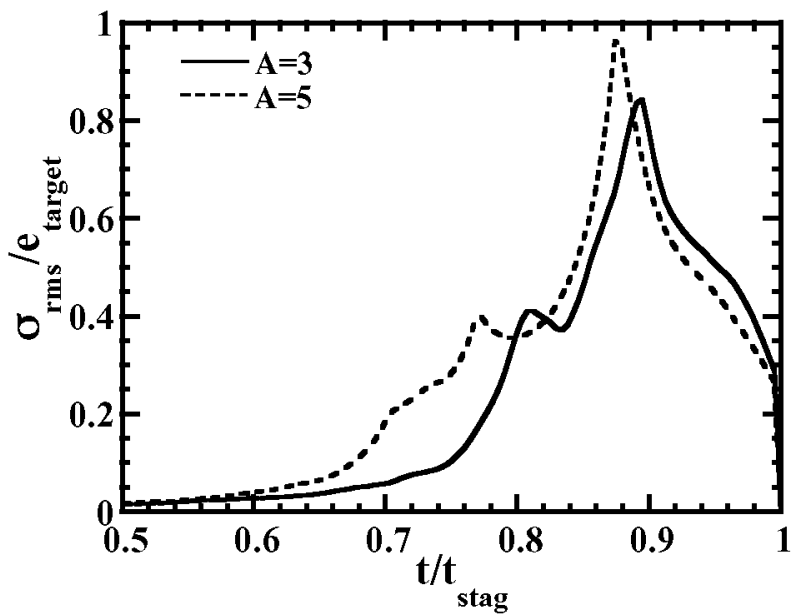

Figure 11. $\sigma_{\text {rms }} / e_{\text {target }}$ versus time renormalized to the end of the laser pulse for $A=3$ and $A=5$ and $v=300 \mathrm{~km} \mathrm{~s}^{-1}$.
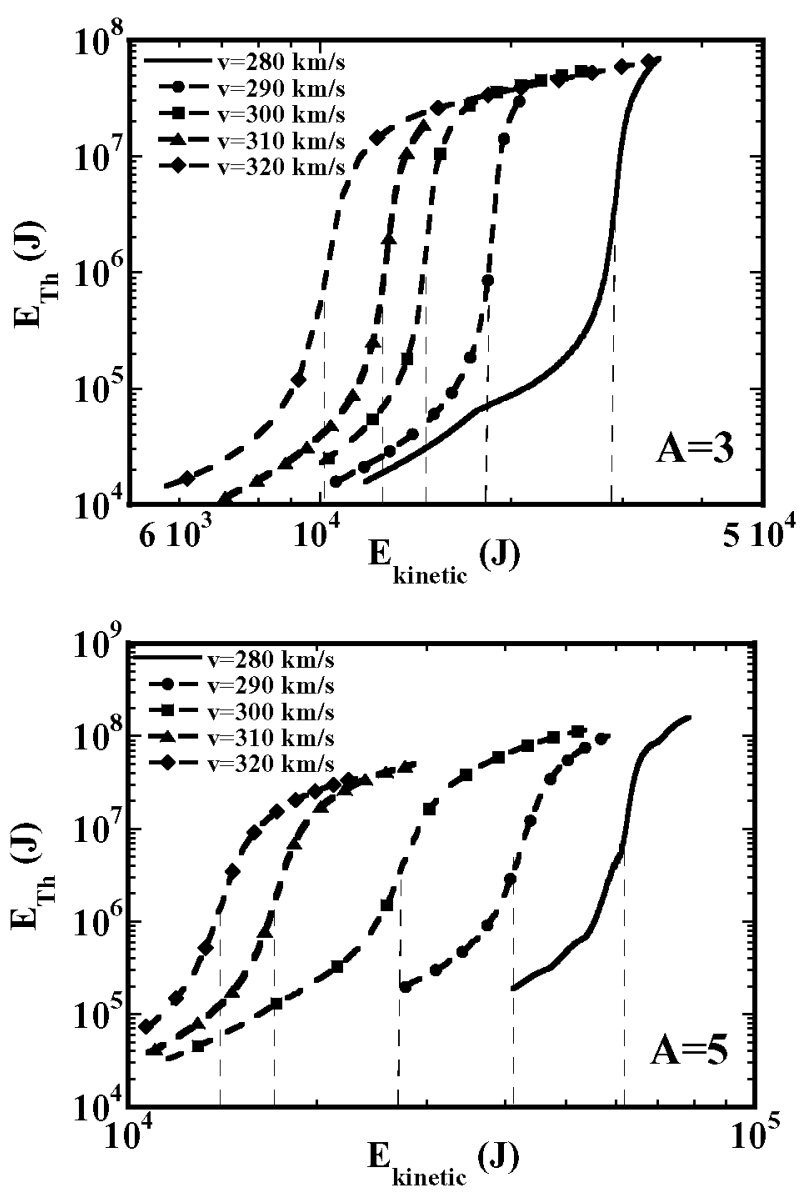

Figure 12. Scaled target families for different implosion velocities for $A=3$ (top) and $A=5$ (bottom).

Each curve is the scaled family of one parent obtained for a given implosion velocity. A transition can be seen between two asymptotic curves defined by the hot-spot and the shell burning energies given by: $E_{i}^{b}[\mathrm{MJ}]=0.33 M_{i}[\mu \mathrm{g}] f_{i}$, with $i \in\{\mathrm{hs}, \mathrm{sh}\}$ where index hs and sh stand for the hot-spot and the shell respectively. the parameters $f_{\mathrm{hs}} \sim 0.5$ and $f_{\mathrm{sh}} \sim 1 /\left(1+70 / \varrho R\left[\mathrm{~kg} \mathrm{~m}^{-2}\right]\right)$ are the burn-up fraction of DT 


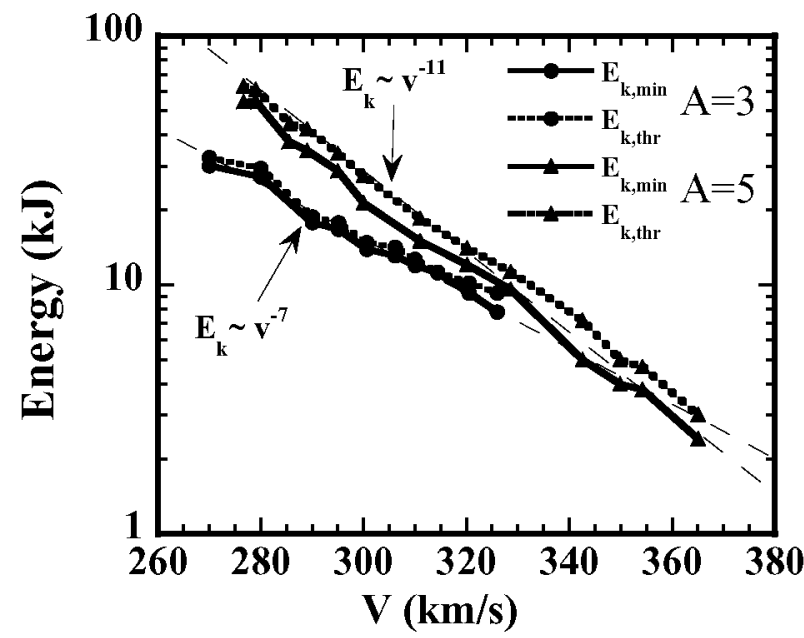

Figure 13. Kinetic energies (threshold $E_{k, \text { thr }}$ and minimum for ignition $E_{k, \min }$ ) versus implosion velocity for $A=3$ (dots) and $A=5$ (triangles). Energies scale as $v^{-7}$ and $v^{-11}$ for $A=3$ and $A=5$ respectively.

in the hot-spot and the shell, respectively. Ignition of the hot spot is always achieved whatever the kinetic energy $[55,110]$. In an asymptotic limit, $f_{\mathrm{sh}} \sim 0.3$.

The transition can be seen as the signature of the breakeven. Below the transition, designs are marginally igniting while above it, ignition produced in the hot spot propagates towards the compressed shell by thermonuclear fusion burn wave. In this situation, shell areal density is high enough to trap fusion products and local ionic temperature increases significantly to sustain thermonuclear reactions. This transition is a self-ignition threshold of kinetic energy $E_{k, \text { thr }}$ in the mass rescaling analysis. In this case, $E_{k, \text { thr }}$ is the kinetic energy maximizing the first derivative of scaling curve: $E_{k, \text { thr }} \in\left\{E_{k} ; \max \left[\mathrm{d}\left(\ln \left(E_{\mathrm{th}}\right)\right) / \mathrm{d} E_{k}\right]\right\}$.

\subsection{Kinetic energy thresholds}

Past works $[97,106,111-118]$ have given scaling laws relating the minimum kinetic energy required for isobaric selfignition to the implosion velocity $v$, the shell adiabat $\alpha_{i f}$, and the peak ablation pressure $p_{a}$ applied at the end of the acceleration stage. An exhaustive description of different scaling is done in [97] where discrepancy is highlighted between models and numerical fits concerning the ignition criteria: $J_{s}=(\varrho R)_{h} T_{h}=$ const or $J_{s} \propto \mathrm{v}$. The self-consistent model developed in [97] integrating the return shock propagation in the shell and the change of shell adiabat at stagnation leads to the following minimum kinetic energy scaling for ignition: $E_{k, \min }=$ $59[\mathrm{~kJ}] \alpha_{\text {if }}^{2.4}\left(\mathrm{v}\left[\mathrm{km} \mathrm{s}^{-1}\right] / 300\right)^{-5}(\mathrm{P}[\mathrm{MBar}])^{-0.39}$, similar to the one used in recent literature [119] and proposed by Hermann et al [117]: $E_{k, \min } \propto \mathrm{v}^{-5.89 \pm 0.12} \alpha_{\mathrm{if}}^{1.88 \pm 0.05} p_{a}^{-0.77 \pm 0.12}$. In these scalings, ignition is not occurring at the same energy than $E_{k, \text { thr }}$ but when target gain $G=E_{\mathrm{Th}} / E_{k, \min }=1$ ( $G$ is defined as the ratio of the capsule thermonuclear yield $E_{\mathrm{Th}}$ over the capsule absorbed energy $E_{k, \min }$ ), achieved at lower kinetic energy than $E_{k \text {,thr }}$. Thus, figure 13 compares these energies for both A.

Despite a shift in amplitude, both quantities, $E_{k, \text { thr }}$ and $E_{k \text {, min }}$, have same tendencies. Nevertheless, it is worth to
Table 2. $\beta$-scaling of ignition energy obtained for different models of literature.

\begin{tabular}{lll}
\hline Model & $A=3$ & $A=5$ \\
\hline Hermann et al $[117]$ & $\beta=-9.6$ & $\beta=-9$ \\
Kemp et al $[118]$ & $\beta=-9.84$ & $\beta=-9.3$ \\
Betti et al $[97]$ & $\beta=-6.87$ & $\beta=-6.6$ \\
\hline
\end{tabular}

notice a significant difference in kinetic energies between initial aspect ratios, especially for low implosion velocities where the kinetic energy for ignition at $A=5$ is the twice of $A=3$. This should have strong implication for shock ignition at low implosion velocities [38]. Power law dependences with velocity are found the same whatever the definition of the energy (threshold or minimum for ignition) but with different exponents between A. Indeed, $E_{k, \text { thr }}$ and $E_{k \text {, min }}$ scale as $v^{-7}$ and $v^{-11}$ for $A=3$ and $A=5$ respectively.

In order to compare our results to existing models, we have also to address the relationship between the maximum ablation pressure $p_{a}$, achieved at the end of the acceleration phase, and the peak implosion velocity. Using our numerical data (especially for the ablation pressure), we get the following scaling: $p_{a} \propto \mathrm{v}^{4.8}$ and $p_{a} \propto \mathrm{v}^{4.1}$ for $A=3$ and 5 respectively. Injecting these lasts in the different scaling of literature, we get $E_{k, \min } \propto \mathrm{v}^{\beta}$ with $\beta$ is given in table 2 .

In complement, we found an ignition criteria $J_{s} \sim$ Const in our calculations. Finally, the adiabat at stagnation is also found $\alpha_{\text {stag }} \propto$ const or $\alpha v^{-0.05}$ very similar to Betti's model $\left(\alpha_{\text {stag }} \propto \mathrm{v}^{-0.67} p_{a}^{0.13} \propto \mathrm{v}^{-0.09 \pm 0.04}\right)$.

To conclude this paragraph, numerical ignition energy scaling departs significantly from the models for both $A$ even if the relationship between peak ablation pressure and peak implosion velocity is accounted for. In addition, we notice that the ignition criteria is not varying with the implosion velocity as the adiabat at stagnation. In fact, the last scales relatively well with the Betti's model [97].

\subsection{Effects of scaling on RTI and TPDI}

We now address the scaling of Rayleigh-Taylor and TwoPlasmons Decay Instabilities when the kinetic energy is rescaled.

4.2.1. Scaling of two-plasmons decay instability In the corona, following the previous assertion, the electronic temperature is not modified by the scaling while $\mathrm{L}_{n e} \sim \xi$. Thus the TPD intensity threshold should vary as: $I^{\prime} 2 \omega_{p}=\xi^{-1} I_{2 \omega_{p}}$. We also assume that kinetic energy scales as $E_{k}{ }^{\prime}=\xi^{3} E_{k}$. Thus, we can predict that the threshold scales as: $I_{2 \omega_{p}} \propto E_{k}^{-1 / 3}$ and that bigger the target, more sensitive to TDP the design is. To check this, we have calculated the TPD intensity threshold versus the kinetic energy for $A=3$ and $A=5$ and the whole set of designs presented in figure 12 . Figure 14 represents the variation of the TPD threshold with $E_{k}$. As it can be seen, scaling is not the same between both $A$. Indeed, $I_{2 \omega_{p}} \propto E_{k}^{-0.3}$ and $E_{k}^{-0.18}$ for $A=3$ and $A=5$ respectively. We find the predicted scaling $\left(\propto E_{k}^{-1 / 3}\right)$ holds only for $A=3$, whilst for $A=5$, it departs from our scaling

In addition, as the absorbed incident laser intensity $I=$ Const versus $\xi$, the ratio $\eta=I / I_{2 \omega_{p}} \propto E_{k}^{0.3}$ and $E_{k}^{0.18}$ 


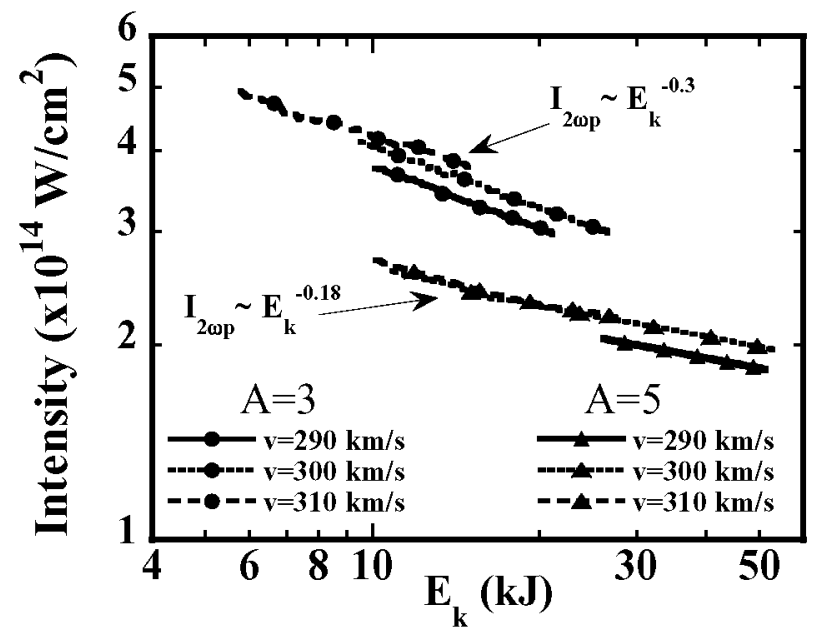

Figure 14. TPD intensity threshold $\left(\times 10^{14} \mathrm{~W} \mathrm{~cm}^{-2}\right)$ versus kinetic energies for different implosion velocities $\left(v=290 \mathrm{~km} \mathrm{~s}^{-1}\right.$ : plain lines; $v=300 \mathrm{~km} \mathrm{~s}^{-1}$ : dotted lines; $v=310 \mathrm{~km} \mathrm{~s}^{-1}$ : long dotted lines) for $A=3$ (circles) and $A=5$ (triangles). Intensities scale as $E_{k}^{-0.18}$ and $E_{k}^{-0.3}$ for $A=3$ and $A=5$ respectively.

for $A=3$ and $A=5$ respectively. We compare the variation of $\eta$ and we observe that while $\eta$ stays in the same amplitude ( $\eta \in[3,4]$ ) for $A=3$ whatever the implosion velocity is, it evolves significantly for $A=5$, between $\eta \in[2.5,3.5]$ for $v=290-300 \mathrm{~km} \mathrm{~s}^{-1}$ until $\eta \in[3,4]$ for $v=310 \mathrm{~km} \mathrm{~s}^{-1}$, the same level than for $A=3$.

However, due to a light dependance in the exponent, $A=5$ families will be less sensitive to TPD while for $A=3$, TPD instability will be be more and more deleterious when kinetic energy will increase. For the last, the consequence should be the increase of the preheating and shell adiabat and the displacement of the ignition threshold towards higher kinetic energies.

4.2.2. Scaling of Rayleigh-Taylor instability Effect of scaling is much more complicated to take into account. Indeed, even though the linear growth rate is scalable, the transition to nonlinear regime introduces an artifact in the scaling. Thus no real tendencies are available. Indeed, all the peak ratios $\sigma_{\mathrm{rms}} / e_{\text {target }}$ evolve in an interval $[0.8,1.1]$ indicating that the shell have a strong probability to disrupt during the acceleration stage.

\section{Conclusion}

We have addressed direct-drive target design with low initial aspect ratios. A first part concerns the definition of the laser pulse and the sensitivity of the design to the random variations of its laser pulse used to implode it. This gives an estimate of the thermonuclear energy delivered at the end of the implosion by fusion process. It is seen that when the laser pulse is randomly varying, a significant modification of the peak implosion velocity leads to a transition towards a production of high energy due to fusion process. The ignition occurs at higher implosion velocities for $\mathrm{A}=5$ than for $\mathrm{A}=3$ indicating that low initial aspect ratio design should be easier to ignite than higher ones. This first conclusion is confirmed when a rescaling is done to find the kinetic energy threshold.
Indeed, $A=3$-designs have lower kinetic energy threshold than $A=5$-designs whatever their implosion velocities.

Our numerical calculations indicate that the ignition threshold scales with power laws significantly different than the ones usually found in literature. These differences are completed by the fact that the $J_{s}$ factor and the adiabat at stagnation are both constant and does not vary with the implosion velocity. This comparison is done by accounting for the dependence in peak ablation pressure of the ignition threshold.

An analysis of robustness regarding two-plasmons decay and Rayleigh-Taylor instabilities is done for the parent designs and for rescaled designs. It is found that the $A=3$-design is much more sensitive to TPD than $A=5$ designs while RTI leads to the contrary. Embedded models dealing with linear and non-linear regime of the RTI show that the shell of $A=5$ designs is systematically broken during the acceleration stage, even for scaled designs. In addition, It is found that rescaling procedure has not real impact on both instabilities. A scaling with kinetic energy is given for the TPD threshold for both A but exponent stays at very low levels.

To finally conclude this work, while looking at low initial aspect ratios was supposed to be more robust regarding RTI, we find that the main interest of reducing the initial aspect ratio stays in the reduction of the ignition velocity and thus the ignition threshold and not in the robustness. Indeed, alternative ways exist to reduce significantly the effect of RTI, such as adiabat shaping or double-front stabilization.

\section{Acknowledgment}

One (BC) of the authors would like to thank L. Masse and P. Gauthier for fruitful discussions.

\section{References}

[1] Lawson J.D. 1957 Proc. Phys. Soc. 706

[2] Anklam T.M., Dunne M., Meier W.R., Powers S. and Simon A. 2011 Fusion Sci. Technol. 6066

[3] Bodner S.E., Schmitt A.J. and Sethian J. 2013 High Pow. Laser Sci. Eng. 12

[4] Amendt P., Dunne M., Ho D. and Lindl J. 2011 Fusion Sci. Technol. 6049

[5] Dunne M., Moses E.I., Amendt P., Anklam T., Bayramian A. and E B. 2011 Fusion Sci. Tech. 6019

[6] Dunne M. 2006 Nature Phys. 22

[7] Moses E.I. 2011 Fusion Sci. Technol. 6011

[8] Lion C. 2010 J. Phys.: Conf. Ser. 244012003

[9] Lindl J. 1995 Phys. Plasmas 23933

[10] Edwards M.J. et al 2013 Phys. Plasmas 20070501

[11] Giorla J. et al 2006 Plasma Phys. Control. Fusion 48 B75

[12] Lindl J. 2004 Phys. Plasmas 11339

[13] Laffite S. and Loiseau P. 2010 Phys. Plasmas 17102704

[14] McCrory R. et al 2007 Eur. Phys. J D 44233

[15] Canaud B., Fortin X., Garaude F., Meyer C., Philippe F., Temporal M., Atzeni S. and Schiavi A. 2004 Nucl. Fusion 441118

[16] Skupsky S. et al 2004 Phys. Plasmas 112763

[17] Radha P.B. et al 2013 Phys. Plasmas 20056306

[18] Canaud B. et al 2007 Plasma Phys. Control. Fusion 49 B601

[19] Canaud B., Garaude F., Clique C., Lecler N., Masson A., Quach R. and Van der Vliet J. 2007 Nucl. Fusion 471652

[20] Canaud B. and Garaude F. 2005 Nucl. Fusion 45 L43

[21] Lehmberg R. and Goldhar J. 1987 Fusion Technol. 11532 
[22] Bodner S.E., Colombant D.G., Schmitt A.J., Gardner J.H., Lehmberg R.H. and Obenschain S.P. 2002 Fusion Eng. Des. 6093

[23] Schmitt A.J., Colombant D.G., Velikovich A.L., Zalesak S.T., Gardner J.H., Fyfe D.E. and Metzler N. 2004 Phys. Plasmas 112716

[24] Schmitt A.J., Bates J.W., Obenschain S.P., Zalesak S.T., Fyfe D.E. and Betti R. 2010 Fusion Sci. Technol. 56377

[25] Temporal M., Canaud B. and LeGarrec B.J. 2010 Phys. Plasmas 17022701

[26] Velarde P., Ogando F., Eliezer S., Martinez-Val J.M., Perlado J.M. and Murakami M. 2005 Laser Part Beams 2343

[27] Murakami M., Nagatomo H., Azechi H., Ogando F., Perlado M. and Eliezer S. 2006 Nucl. Fusion 4699

[28] Murakami M. and Nagatomo H. 2005 Nucl. Instrum. Methods A $\mathbf{5 4 4} 67$

[29] Tabak M., Hammer J., Glinsky M., Kruer W., Wilks S., Woodworth J., Campbell E. and Perry M. 1994 Phys. Plasmas 11626

[30] Roth M. et al 2001 Phys. Rev. Lett. 86436

[31] Atzeni S., Schiavi A. and Bellei C. 2007 Phys. Plasmas 14052702

[32] Hallo L., Olazabal-Loume M., Ribeyre X., Drean V., Schurtz G., Feugeas J., Breil J., Nicolai P. and Maire P. 2009 Plasma Phys. Contr. Fusion 51014001

[33] Betti R. and Zhou C. 2005 Phys. Plasmas 12110702

[34] Zhou C.D. and Betti R. 2007 Phys. Plasmas 14072703

[35] Ribeyre X., Nicolai P., Schurtz G., Olazabal-Loumé M., Breil J., Maire P.H., Feuges J.L., Hallo L. and Tikhonchuk V.T. 2008 Plasma Phys. Control. Fusion 50025007

[36] Atzeni S. 1999 Phys. Plasmas 63316

[37] Betti R., Zhou C.D., Anderson K., Perkins L.J., Theobald W. and Solodov A.A. 2007 Phys. Rev. Lett. 98155001

[38] Canaud B. and Temporal M. 2010 New J. Phys. 12043037

[39] Ribeyre X., Schurtz G., Lafon M., Galera S. and Weber S. 2009 Plasma Phys. Control. Fusion 51015013

[40] Perkins L.J., Betti R., Lafortune K.N. and Williams W.H. 2009 Phys. Rev. Lett. 103045004

[41] Schmitt A.J., Bates J.W., Obenschain S.P., Zalesak S.T. and Fyfe D.E. 2010 Phys. Plasmas. 17042701

[42] Lafon M., Ribeyre X. and Schurtz G. 2010 Phys. Plasmas 17052704

[43] Atzeni S. et al 2009 Nucl. Fusion 49055008

[44] Atzeni S. and Temporal M. 2003 Phys. Rev. E 671433

[45] Temporal M., Jaouen S., Masse L. and Canaud B. 2006 Phys. Plasmas 13122701

[46] Sanz J., Garnier J., Cherfils-Clerouin C., Canaud B. Masse L. and Temporal M. 2005 Phys. Plasmas 12112702

[47] Fan Z., Zhu F., Pei W., Ye W., Li M., Xu X., Wu J., Dai Z. and Wang L. 2011 Eur. Phys. Lett. 9965003

[48] Temporal M. and Canaud B. 2009 Eur. Phys. J. D 55139

[49] Temporal M., Ramis R., Canaud B., Brandon V., Laffite S. and Garrec B.J.L. 2011 Plasma Phys. Control. Fusion 53124008

[50] Temporal M., Canaud B., Garbett W.J. and Ramis R. 2014 Phys. Plasmas 20012710

[51] Canaud B., Temporal M. and Laffite S. 2012 Laser Part Beams 30183

[52] Betti R. et al 2008 J. Phys. Conf. Ser. 112022024

[53] Depierreux S. et al 2011 Plasma Phys. Control. Fusion 53124034

[54] Bell T. and Tzoufras M. 2011 Plasma Phys. Control. Fusion 53045010

[55] Canaud B., Laffite S. and Temporal M. 2011 Nucl. Fusion 51062001

[56] Bodner S.E. et al 1998 Phys. Plasmas 51901

[57] Sacks R. and Darling D. 1987 Nucl. Fusion 27447

[58] Canaud B., Fortin X., Garaude F., Meyer C. and Philippe F. 2004 Laser Part Beams 22109
[59] Collins T.J.B., Marozas J., Betti R., Harding D., McKenty P.W., Radha P.B., Skupsky S., Goncharov V.N., Knauer J.P. and McCrory R.L. 2007 Phys. Plasmas 14056308

[60] Philippe F., Canaud B., Fortin X., Garaude F. and Jourdren H. 2004 Laser Part Beams 22171

[61] Hazak G., Velikovich A.L., Gardner J.H. and Dahlburg J.P. 1998 Phys. Plasmas 54357

[62] Collins T.J.B., Poludnenko A., Cunningham A. and Frank A. 2005 Phys. Plasmas 12062705

[63] Elbaz D., Dias F., Canaud B. and Ballereau P. 2010 Phys. Plasmas 17012702

[64] Elbaz D., Canaud B., Ballereau P. and Dias F. 2013 Shock Waves 2381

[65] Poludnenko A.Y., Frank A. and Blackman E.G. 2002 Astrophys. J. $\mathbf{5 7 6} 832$

[66] Poludnenko A.Y., Dannenberg K.K., Drake R.P., Frank A. Knauer J., Meyerhofer D.D., Furnish M., Asay J.R. and Mitran S. 2004 Astrophys. J. 604213

[67] Piron R., Ballereau P. and Canaud B. 2009 Eur. J. Mech. B: Fluids 28613

[68] Elbaz D., Jourdan G., Houas L., Jaouen S., Ballereau P., Dias F. and Canaud B. 2012 Phys. Rev. E 85066307

[69] Wouchuk J.G., Huete Ruiz de Lira C. and Velikovich A.L. 2009 Phys. Rev. E 79066315

[70] Huete Ruiz de Lira C., Velikovich A.L. and Wouchuk J.G. 2011 Phys. Rev. E 83056320

[71] Huete Ruiz de Lira C., Wouchuk J.G., Canaud B. and Velikovich A.L. 2012 J. Fluid. Mech. 700214

[72] Velikovich A.L., Huete Ruiz de Lira C. and Wouchuk J.G. 2012 Phys. Rev. E 85016301

[73] Takabe H., Mima K., Montierth L. and Morse R. 1985 Phys. Fluids 283676

[74] Goncharov V.N., Betti R., McCrory R.L., Sorotokin P. and Verdon C. 1996 Phys. Plasmas 31402

[75] Betti R., Goncharov V.N., McCrory R.L. and Verdon C. 1998 Phys. Plasmas 51446

[76] Goncharov V.N., McKenty P.W., Skupsky S., Betti R., McCrory R.L. and Cherfils-Clérouin C. 2000 Phys. Plasmas 75118

[77] Anderson K. and Betti R. 2003 Phys. Plasmas 104448

[78] Goncharov V.N., Knauer J.P., McKenty P.W., Radha P.B., Sangster T., Skupsky S., Betti R., McCrory R.L. and Meyerhofer D.D. 2003 Phys. Plasmas 101906

[79] Anderson K. and Betti R. 2004 Phys. Plasmas 115

[80] Collins T.J.B., Knauer J.P., Betti R., Boehly T., Delttrez J.A., Goncharov V.N., Meyerhofer D.D., McKenty P.W. and Skupsky S. 2004 Phys. Plasmas 111569

[81] Fujioka S. et al 2004 Phys. Rev. Lett. 92195001

[82] Fujioka S. et al 2004 Phys. Plasmas 112814

[83] McKenty P.W., Goncharov V.N., Town R., Skupsky S. and Betti R. 2001 Phys. Plasmas 82315

[84] Simon A., Short R.W., Williams E.A. and Dewandre T. 1983 Phys. Fluids 263107

[85] Afeyan B.B. and Williams E.A. 1997 Phys. Plasmas 43827

[86] Rayleigh L. 1900 Sci. Pap. Camb. England II 200

[87] Taylor G. 1959 Proc. R Soc. London A 201192

[88] Haan S. 1989 Phys. Rev. A 395812

[89] Buresi E., Coutant J. and Dautray R. 1986 Laser Part Beams 4531

[90] Lyon S.P. and Johnson J.D. (ed) 1992 Sesame: the lanl equation of state database Report number LA-UR-92-3407 (Los Alamos, NM: Los Alamos National Laboratory)

[91] Caillabet L., Mazevet S. and Loubeyre P. 2011 Phys. Rev. B 83094101

[92] Caillabet L., Canaud B., Salin G., Mazevet S. and Loubeyre P. 2011 Phys. Rev. Lett. 107115004

[93] Hubbard W.B. 1966 Astrophys. J 146858

[94] Recoules V., Lambert F., Decoster A., Canaud B. and Clerouin J. 2009 Phys. Rev. Lett. 102075002

[95] Kidder R. 1976 Nucl. Fusion 163

[96] Brandon V., Canaud B., Laffite S., Temporal M. and 
Ramis R. 2013 Laser Pant Beams 31141

[97] Betti R., Anderson K., Goncharov V.N., McCrory R.L., Meyerhofer D.D., Skupsky S. and Town R. 2002 Phys. Plasmas 92277

[98] Fraley G., Linnebur E., Mason R. and Morse R. 1974 Phys. Fluids 17474

[99] Froula D. et al 2012 Phys. Rev. Lett. 108165003

[100] Michel D.T., Maximov A., Short R., Hu S., Myatt J., Seka W., Sodolov A., Yaakobi B. and Froula D. 2012 Phys. Rev. Lett. 109155007

[101] Bodner S.E. 1974 Phys. Rev. Lett. 33761

[102] Piriz A.R., Sanz J. and Iba nez L.F. 1997 Phys. Plasmas 41117

[103] Betti R., McCrory R.L. and Verdon C.P. 1993 Phys. Rev. Lett. 713131

[104] Betti R., Goncharov V.N., McCrory R.L. and Verdon C. 1995 Phys. Plasmas 23844

[105] Legay G. 2013 CHSi power spectrum: effect of smoothing technics private communication

[106] Basko M. and Johner J. 1998 Nucl. Fusion 381779

[107] Murakami M. and Iida S. 2002 Phys. Plasmas 92745
[108] Falize E., Bouquet S. and Michaut C. 2009 Astrophys. Space Sci. 322107

[109] Falize E., Michaut C. and Bouquet S. 2011 Astrophys. J. 73096

[110] Amendt P., Colvin J., Tipton R., Hinkel D., Edwards M. Landen O., Ramshaw J., Suter L., Varnum W. and Watt R. 2002 Phys. Plasmas 92221

[111] Meyer-ter-Vehn J. 1982 Nucl. Fusion 22561

[112] Basko M. 1995 Nucl. Fusion 3587

[113] Piriz A.R. 1996 Fusion Eng. Des. 32 \& 33561

[114] Levedhal W. and Lindl J. 1997 Nucl. Fusion 37165

[115] Lobatchev V. and Betti R. 2000 Phys. Rev. Lett. 854522

[116] Herrmann M., Tabak M. and Lindl J. 2001 Nucl. Fusion 4199

[117] Herrmann M., Tabak M. and Lindl J. 2001 Phys. Plasmas 82296

[118] Kemp A., Meyer-ter-Vehn J. and Atzeni S. 2001 Phys. Rev. Lett. 863336

[119] Atzeni S., Marocchino A., Schiavi A. and Schurtz G. 2013 New J. Phys. 15045004 